\title{
Biocompatible Fluorescent Probe with the Aggregation-induced Emission Characteristic for Live Cell Imaging
}

\author{
Yanan $\mathrm{XI}^{1, \mathrm{a}}$, Wei GAO ${ }^{1, \mathrm{~b}}$, Jingtian $\mathrm{CHEN}^{1, \mathrm{c}}$, Shuchen $\mathrm{ZHANG}^{1, \mathrm{~d}}$,Tao YANG ${ }^{1,2, \mathrm{e}, \text { and }}$ \\ Baoxiang $\mathrm{GAO}^{1,2,3, \mathrm{f}, *}$ \\ ${ }^{1}$ College of Chemistry and Environmental Science, Hebei University, Baoding, 071002, P. R. China \\ ${ }^{2}$ Key Laboratory of Medicinal Chemistry and Molecular Diagnosis of Ministry of Education, Hebei \\ University, Baoding, 071002, P. R. China \\ ${ }^{3}$ Key Laboratory of Analytical Science and Technology of Hebei Province, Hebei University, \\ Baoding, 071002, P. R. China \\ a15233749702@163.com, ${ }^{\mathrm{b}} 15733205590 @ 163 . c o m,{ }^{\mathrm{c}} \mathrm{chenjingtian37@163.com,}$ \\ d15100270883@163.com, ${ }^{\mathrm{e}}$ tyang@hbu.edu.cn, ${ }^{\mathrm{f}}$ bxgao@hbu.edu.cn
}

\begin{abstract}
A new kind of biocompatible probe (PEG-TPEFE) with aggregation-induced emission (AIE) characteristic was reported, which was synthesized from fluorene, tetraphenylethylene (TPE) and polyethylene glycol monomethyle ether $1000\left(\mathrm{PEG}_{1000}\right)$. PEGTPEFE can aggregate into nanosphere and emit strong blue fluorescence in aqueous media. Cytotoxicity assay reveals low cytotoxicity and excellent biocompatibility of this nanoprobe. This nanoprobe was internalized and accumulated by live cells and shown high photostability.
\end{abstract}

Keywords: Fluorescent probe, aggregation-induced emission (AIE), photophysical properties, cellular imaging

\section{Introduction}

Fluorescence bio-imaging technique has been proven to be useful in biology research and clinical diagnosis, which benefits high sensitivity, fine temporal and spatial resolution, easy operation, and cost-effectiveness. ${ }^{[1]}$ Furthermore, fluorescence bio-imaging provided important information about biological and biochemical processes, such as cell transplantation, division, migration, fusion, and lysis. ${ }^{[2]}$ There are several characteristics of successful fluorescent probes for bio-imaging including water solubility, brightness, low cytotoxicity, and photostability. Organic fluorophores are widely used as probes for fluorescence bio-imaging. ${ }^{[3]}$ However, conventional organic fluorophores exhibit poor water solubility and very weak fluorescence in water because of aggregation-caused quenching (ACQ) ${ }^{[4]}$ Recently, Tang and co-workers have developed a novel class of organic fluorophores with a unique aggregation-induced emission (AIE) characteristics,

\footnotetext{
* Corresponding author: bxgao@hbu.edu.cn
} 
which is exactly opposite to the ACQ effect for conventional organic fluorophores. ${ }^{[5]}$ Based on the virtue of the AIE phemomenon, the fluorophores with AIE characteristics are attracting increasing attention on sensing and imaging applications. ${ }^{[6]}$ Additionally, fluorescent probes with low cytotoxicity are highly desired. Poly(ethylene glycol) (PEG) is a water-soluble polymer, which is widely used in the biomedical field of drug delivery currently, and the only polymeric therapeutic that has market approval for different drugs. ${ }^{[7]}$ Very recently, our group and others reported the design and synthesis of PEGfunctionalized organic fluorophores for bio-imaging applications. ${ }^{[8,9]}$

Our interest is to develop biocompatible dyes with high fluorescence in aqueous solutions for bioimaging. In this study, we reported a novel fluorescence probe with the AIE property, in which polyethylene glycol (PEG) was attached to the fluorophore to improve biocompatibility.

\section{Results and Discussions}

The synthesis route of PEG-TPEFE was described in Scheme 1. 2,7-Bis(4,4,5,5tetramethyl-1,3,2-dioxaborolan-2-yl)-9,9-bis(3-tert-butylpropanoate))fluorene 1, the key intermediate for the synthesis of PEG-TPEFE, was obtained according to the literature procedures. ${ }^{[10]}$ TPEFE ester was obtained by Suzuki coupling of 2,7-Bis(4,4,5,5tetramethyl-1,3,2-dioxaborolan-2-yl)-9,9-bis(3-tert-butylpropanoate)fluorene $\mathbf{1}$ with 6 bromo- 1,1',1",1'"-ethene-1,1,2,2-tetrayltetrabenzene 2.TPEFE COOH was obtained by TPEFE ester dissolved in DCM and treated with $\mathrm{CF}_{3} \mathrm{COOH}$ at room temperature for $24 \mathrm{~h}$. PEG-TPEFE was obtained by TPEFE COOH and PEG under the catalyst system of TBTU and DBU in the solution of DMF at room temperature for $32 \mathrm{~h}^{[11]}$ and then purified by recycling preparative chromatogram. All compounds were characterized by NMR spectroscopy and matrix-assisted laser desorption/ionization mass spectrometry. The characteristics of PEG-TPEFEwere described in Figure 1.

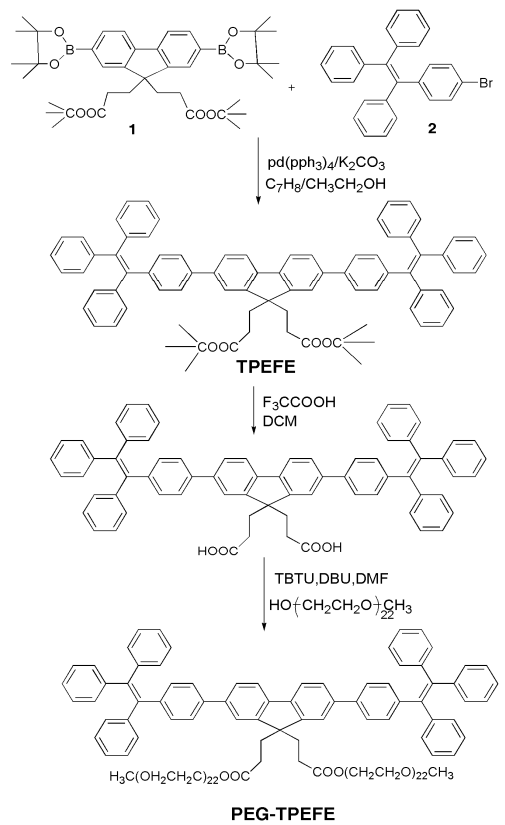

Scheme 1. The synthesis of PEG-TPEFE 

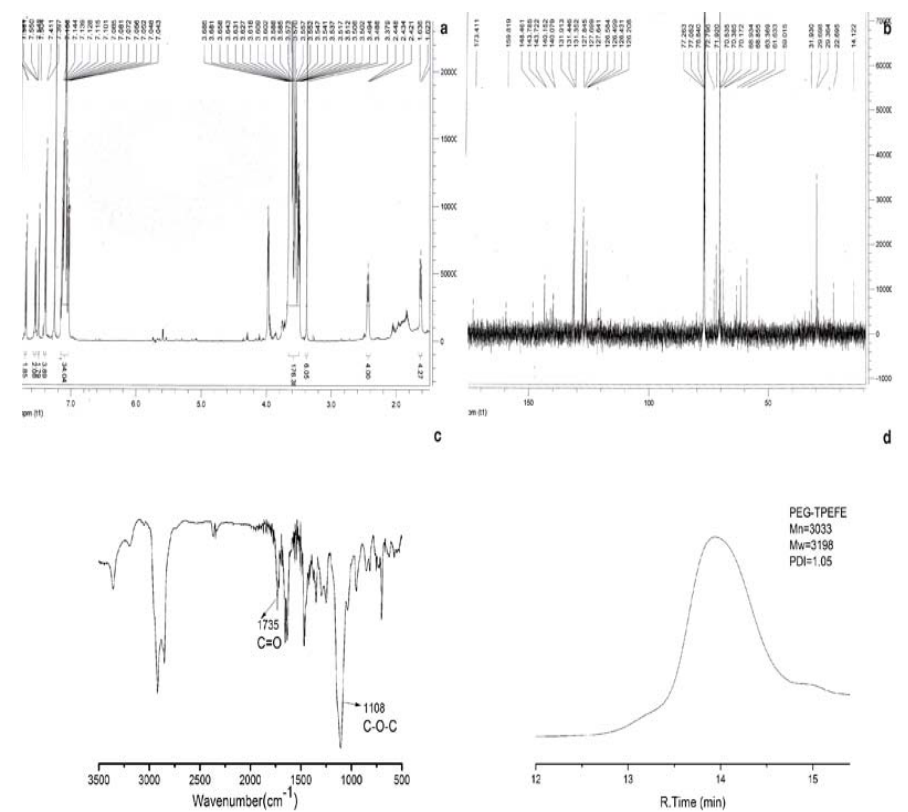

Figure 1. (a) ${ }^{1} \mathrm{H}$ NMR spectrum of Compound PEG-TPEFE $\left(\mathrm{CDCl}_{3}, 600 \mathrm{MHz}\right)$. (b) ${ }^{1} \mathrm{C}$ NMR spectrum of Compound PEG-TPEFE $\left(\mathrm{CDCl}_{3}, 600 \mathrm{MHz}\right)$. (c) IR spectrum of Compound PEG-TPEFE. (d) GPC chromatograms of Compound PEG-TPEFE
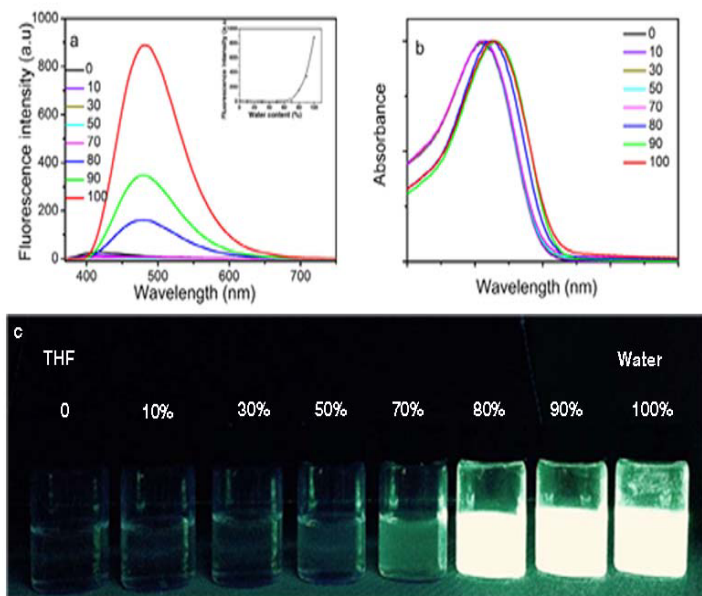

Figure 2. (a) Fluorescence spectra of TPE-FEPEG in Water/THF system with different water contents ([TPE-FEPEG] $=100 \mu \mathrm{g} / \mathrm{mL}$, excitation wavelength: $360 \mathrm{~nm}$ ). Insert: change of fluorescent intensity of TPE-FEPEG in Water/THF system at $480 \mathrm{~nm}$. (b) UVVis absorption spectra of TPE-FEPEG in Water/THF system with different water contents.

(c) Photographs of PEG-TPEFE in Water/THF systems with different water contents under UV light (365 $\mathrm{nm})$ 

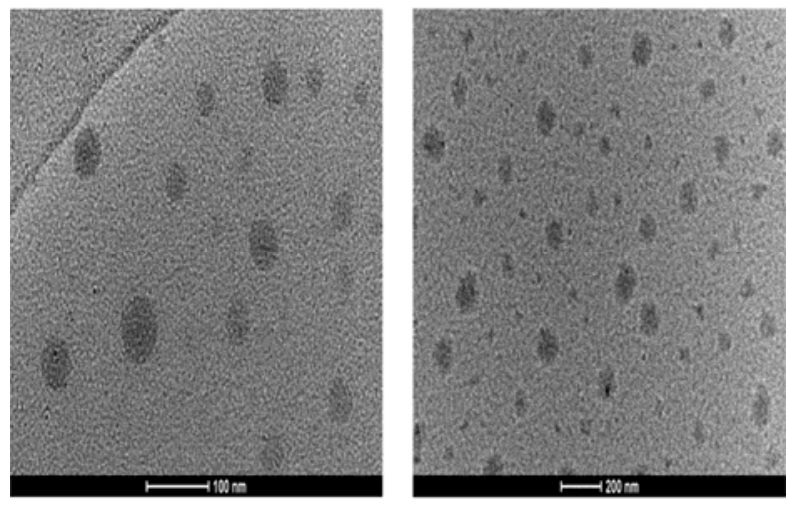

Figure 3. TEM images of PEG-TPEFE

We studied the fluorescence behavior of PEG-TPEFE in Water/THF system. The results as shown in Figure 2a, in dilute THF solution, PEG-TPEFE shows very weak fluorescence with a emission maximum at $420 \mathrm{~nm}$. When the water content increases from 0 to $80 \%$, the fluorescence spectra of the solutions have very small changes. When the water content is $80 \%$ or higher, the fluorescence intensity dramatically enhanced and the emission maximum red shifts to $480 \mathrm{~nm}$. The PEG-TPEFE is non-emissive when the water content less than $80 \%$, but the emission becomes visible when water above $80 \%$ at $480 \mathrm{~nm}$ (insert in Figure 2a). The enhancement is ascribed to aggregate formation, which is induced by the addition of water. The aggregation behavior is also demonstrated by absorption spectra, as shown in Figure 2b, when the water content changes from 0 to $100 \%$, the absorption maximum red shifts from $356 \mathrm{~nm}$ to $363 \mathrm{~nm}$. The Figure $2 \mathrm{c}$ shows clear blue emission from the PEG-TPEFE aggregates under illumination of UV light $(365 \mathrm{~nm})$. These results indicate that PEG-TPEFE is a typical AIE molecule. We ascribe this AIE phenomenon to the rotating units of phenyl rings in the TPE units. The rotor-containing fluorogens of PEG-TPEFE undergo low-frequency motions in dilute solutions, causing fast nonradiative decay of the excited states that makes the fluorogens nonemissive. In the aggregates, these motions are blocked by intermolecular steric interaction, which opens the radiative pathway. ${ }^{[12]}$ Restriction of intramolecular rotation (RIR) is the main reason of TPE for AIE effect as reported. ${ }^{[13]}$ It can be confirmed from the transmission electron microscopy (TEM) images as shown in Figure 3, which indicate that when PEG-TPEFE is dissolved in water, it integrate into nanoparticle with the size remained at 25-100 nm and has a spherical shape.

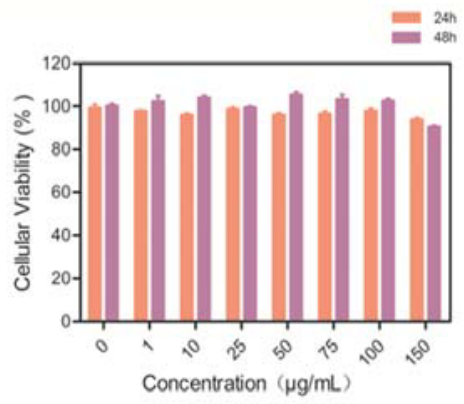

Figure 4. Cell viability of HeLa cells after incubation with different concentration of PEG-TPEFE for 24 and $48 \mathrm{~h}$ 

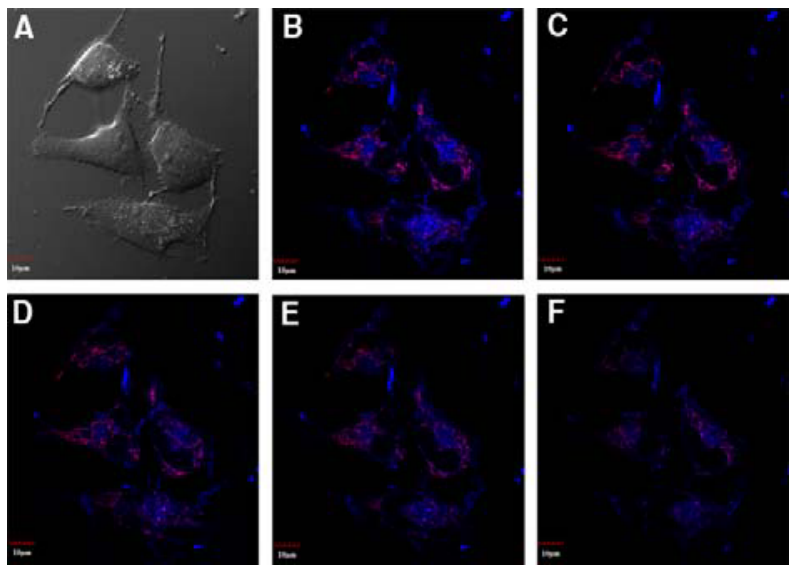

Figure 5. The bright-field images (A) and the overlap images of HeLa cells stained by PEG-TPEFE and Mitotracker Red with continuous 405 and $559 \mathrm{~nm}$ laser scan 0 min (B), 3 $\min (\mathrm{C}), 6 \min (\mathrm{D}), 9 \mathrm{~min}(\mathrm{E})$ and $12 \mathrm{~min}(\mathrm{~F})$. (blue: PEG-TPEFE; magenta: overlap of

PEG-TPEFE and Mitotracker Red )
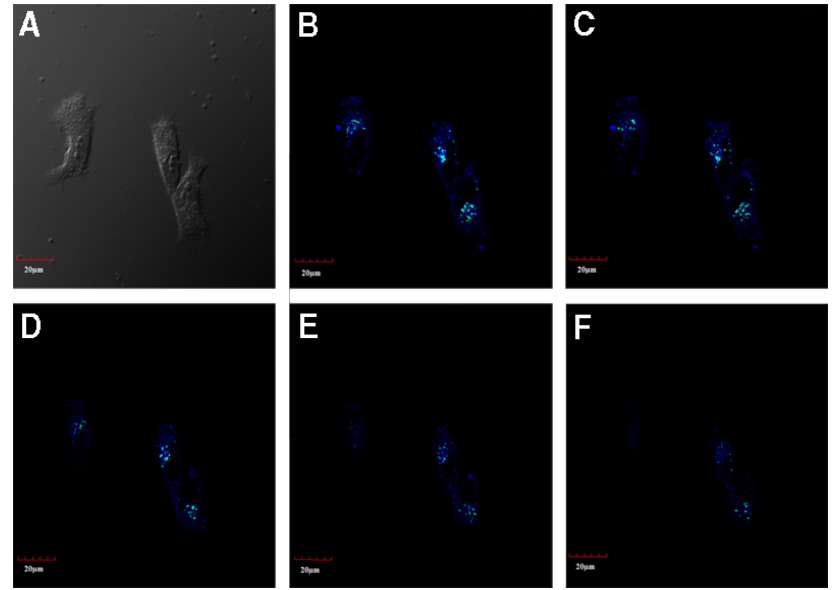

Figure 6. The bright-field images (A) and the overlap images of HeLa cells stained by PEG-TPEFE and Lysotracker Green with continuous 405 and $488 \mathrm{~nm}$ laser scan 0 min (B),

$3 \mathrm{~min}$ (C), $6 \mathrm{~min}$ (D), $9 \mathrm{~min}$ (E) and $12 \mathrm{~min}$ (F). (blue: PEG-TPEFE; cyan: overlap of PEG-TPEFE and Lysotracker Green) 

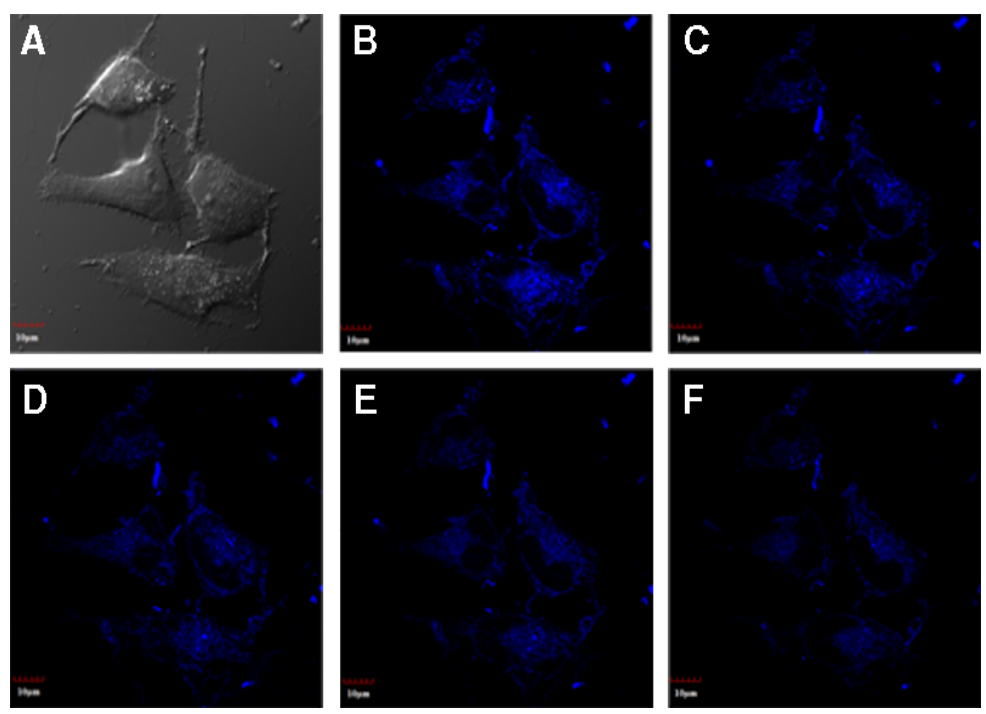

Figure 7. The bright-field images (A) and the confocal fluorescence images of HeLa cells stained by PEG-TPEFE with continuous $405 \mathrm{~nm}$ laser scan 0 min (B), 3 min (C), 6 min (D), $9 \mathrm{~min}(\mathrm{E})$ and $12 \mathrm{~min}(\mathrm{~F})$

To demonstrate the potential utility of the PEG-TPEFE probe for cellular imaging, its cytocompatibility was evaluated by Counting Kit-8 (CCK8) assay in HeLa cells. ${ }^{[14]}$ Figure 4 summarizes the viability of HeLa cells after being cultured with PEG-TPEFE at different concentrations. The results show very low cytotoxicity within $24 \mathrm{~h}$ of incubation time at the concentration of $150 \mu \mathrm{g} / \mathrm{mL}$. The viability basically unchanged even the incubation time extended to $48 \mathrm{~h}$ (all above 90\% viability), which indicating that PEG-TPEFE has low cytotoxicity or excellent biocompatibility. The exceptionally low cytotoxicity is attributed to the PEG chains, which protect the probe from nonspecifically interacting with the extracellular proteins. ${ }^{[15]}$ The low cytotoxicity makes the PEG-TPEFE promising for bioimaging applications.

The application of PEG-TPEFE for cellular imaging was studied by confocal laser scanning microscopy (CLSM). HeLa cells were incubated with PEG-TPEFE $(100 \mu \mathrm{g} / \mathrm{mL})$ for $2 \mathrm{~h}$, the excitation wavelength was fixed at $405 \mathrm{~nm}$ and fluorescent signals were collected from $450 \mathrm{~nm}$ to $550 \mathrm{~nm}$, the fluorescence imagines of the cells were captured. The CLSM images show strong fluorescence from the cells, and indicate that PEG-TPEFE efficiently permeated the cells and accumulated in cell organelles and cytoplasm. To evaluate whether or not the PEG-TPEFE probe is the specific cellular target, HeLa cells were co-stained with PEG-TPEFE, Mitotracker Red (a commercially available mitochondrion-targeting dye) and Lysotracker Green (a commercially available lysosometargeting dye), respectively. The two-color colocalization results revealed that no significant co-localization was found for PEG-TPEFE and Mitotracker Red (Figure 5), indicating the PEG-TPEFE probe is not mitochondrion-targeting dye. In contrast, the majority of PEG-TPEFE probe was distributed in the lysosomes and the cytoplasm (Figure 6), indicating that these PEG-TPEFE have escaped from the endosomes and are released into the cytoplasm. ${ }^{[16]}$

To identify the photostability of PEG-TPEFE in harsh physiological environment, the fluorescence imagines of the cells with continuous laser scanning were captured. After irradiation for $9 \mathrm{~min}$, the intensity of the fluorescence images still remained strong (Figure 7E). These results proved the relatively high photostability of PEG-TPEFE in harsh 
physiological environment. Thus, the highly fluorescent PEG-TPEFE have potential applications as probes for live cell imaging.

\section{Conclusion}

New biocompatible nanoprobe with aggregation-induced emission (AIE) characteristic was designed and synthesized. The PEG-TPEFE can aggregate into nanosphere and emit strong blue fluorescence in aqueous media. Low cytotoxicity, excellent biocompatibility and high photostability make this PEG-TPEFE dye excellent probe for live-cell imaging.

\section{References}

1. J. R. Swedlow, I. Goldberg, E. Brauner, P. K. Sorger, Informatics and quantitative analysis in biological imaging, Science. 300 (2003) 100-102. (b) C. Zhu, L. Liu, Q. Yang, F. Lv, S. Wang, Water-soluble conjugated polymers for imaging, diagnosis, and therapy, Chem. Rev. 112 (2012) 4687-4735. (c) K. Li, B. Liu, Polymer encapsulated conjugated polymer nanoparticles for fluorescence bioimaging, J. Mater. Chem. 22 (2012) 1257-1264.

2. R. M. Hoffman, The multiple uses of fluorescent proteins to visualize cancer in vivo, Nat. Rev. Cancer. 5 (2005) 796-806. (b) N. M. Idris, Z. Q. Li, L. Ye, et al. Stem sell tracking with optically active nanoparticles, Biomaterials. 30 (2009) 5104-5113. (c) J. H. Kim, Y. S. Kim, K. Park, et al, Antitumor efficacy of cisplatin-loaded glycol chitosan nanoparticles in tumor-bearing mice, J. Controlled Release. 127 (2008) 41-49.

3. K. Yang, S. Zhang, G. X. Zhong, et al. Graphene in mice: ultrahigh in vivo tumor uptake and efficient photothermal therapy, Nano Lett. 10 (2010) 3318-3323. (b) J. K. Jaiswal, H. Mattoussi, J. M. Mauro, et al. Long-term multiple color imaging of live cells using quantum dot bioconjugates, Nat. Biotechnol. 21 (2003) 47-51.

4. J. B. Birks, Photophysics of Aromatic Molecules, Wiley, London, 1970. (b) S. A. Jenekhe, J. A. Osaheni, Excimers and exciplexes of conjugated polymers, Science. 265 (1994) 765-768.

5. Y. N. Hong, J. W. Y. Lam, B. Z. Tang, Aggregation-induced emission, Chem. Soc. Rev. 40 (2011) 5361-5388. (b) Y. Yu, C. Feng, Y. Hong, et al. Cytophilic fluorescent bioprobes for long-term cell tracking, Adv. Mater. 23 (2011) 3298-3302. (c) Y. Liu, C. M. Deng, L. Tang, et al. Specific detection of d-glucose by tetraphenylethene-based fluorescent sensor, J. Am. Chem. Soc. 133 (2011) 660-663. (d) M. Li, Y. N. Hong, Z. $\mathrm{K}$. Wang, et al. Fabrication of chitosan nanoparticles with aggregation-induced emission characteristics and their applications in long-term live cell imaging, Macromol. Rapid Commun. 34 (2013) 767-771.

6. T. Sanji, K. Shiraishi, M. Nakamura, M. Tanaka, Fluorescence turn-on sensing of lectins with mannose-substituted tetraphenylethenes based on aggregation-induced emission, J. Chem. Asian. 5 (2010) 817-824. (b) G. X. Feng, C. Y. Tay, Q. X. Chui, et al. Ultrabright organic dots with aggregation-induced emission characteristics for cell tracking, Biomaterials. 35 (2014) 8669-8677

7. M. C. Woodle, Controlling liposome blood clearance by surfance grafted polymers, Adv. Drug Delivery Rev. 32 (1998) 139-152. (b) J. M. Harris, S. Zaplisky, Poly(ethylene glycol): chemistry and biomedical applications, American Chemical Society, Washington DC, 1997. 
8. B. X. Gao, H. X. Li, H. M. Liu, et al. Water-soluble and fluorescent dendritic perylene bisimides for live-cell imaging, Chem. Commun. 47 (2011) 3894-3896. (b) H. M. Liu, Y. L. Wang, C. H. Liu, et al. Fluorescent water-soluble probes based on dendritic PEG substituted perylene bisimides: synthesis, photophysical properties, and live cell images, J. Mater. Chem. 22 (2012) 6176-6181. (c) L. B. Bai, W. Li, J. T. Chen, et al. Water-soluble fluorescent probes based on dendronized polyfluorenes for cell imaging, Macromolecular. Rapid Commun. 34 (2013) 539-547.

9. Li. K. Kai, W. Qin, D. Ding, et al. Polycationic adamantane-based dendrons of different generations display high cellular uptake without triggering cytotoxicity, J. Am. Chem. Soc. 136 (2014) 810-819.

10. T. Ishiyama, Y. Itoh, T. Kitano, N. Miyaura, Synthesis of arylboronates via the palladium(0)-catalyzed cross-coupling reaction of tetra(alkoxo)diborons with aryl triflates, Tetrahedron Lett. 38 (1997) 3447-3450.

11. Tean-d' Amour K. Twibanire and T. Bruce Grindley, Efficient and controllably selective preparation of esters using uronium-based coupling agents, Organic letters. 13 (2011) 2988-2911.

12. J. W. Barr, T. W. Bell, V. J. Catalano, et al. Syntheses, structures, and photoisomerization of (E)- and (Z)-2-tert-butyl-9-(2,2,2-triphenylethylidene)fluorene, J. Phys. Chem. A. 109 (2005) 11650-11654.

13. Q. Zhao, et al. Tetraphenylethenyl-modified perylene bisimide: aggregation-induced red emission, electrochemical property and ordered microstructures, J. Mater. Chem. 22 (2012) 7387-7394. (b) Q. Peng, Y. Yi, Z. Shuai, J. Shao, Toward quantitative prediction of molecular fuorescence quantum efficiency: role of duschinsky rotation, J. Am. Chem. Soc. 129 (2007) 9333-9339.

14. X. W. Long, Zh. H. Zhang, Sh. C. Han, et al. Structural mediation on polycation nanoparticles by sulfadiazine to enhance DNA transfection efficiency and reduce toxicity, ACS Appl. Master. interfaces. 7 (2015) 7542-7551.

15. J. M. Harris and R. B. Chess, Effect of pegylation on pharmaceutical, Nat. Rev. Drug Discovery. 2 (2003) 214-221.

16. H. Huang, B. Yu, P. Zhang, et al. Highly charged ruthenium(II) polypyridyl complexes as lysosome-localized photosensitizers for two-photon photodynamic therapy, Angew. Chem. Int. Ed. 127 (2015) 14255-14258. 